\title{
CLASSIFICATION OF NEARLY INVARIANT SUBSPACES OF THE BACKWARD SHIFT
}

\author{
ERIC HAYASHI
}

(Communicated by Paul S. Muhly)

\begin{abstract}
Let $S^{*}$ denote the backward shift operator on the Hardy space $H^{2}$ of the unit disk. A subspace $M$ of $H^{2}$ is called nearly invariant if $S^{*} h$ is in $M$ whenever $h$ belongs to $M$ and $h(0)=0$. In particular, the kernel of every Toeplitz operator is nearly invariant. A function theoretic characterization is given of those nearly invariant subspaces which are the kernels of Toeplitz operators, and it is shown that they can be put into one-to-one correspondence with the Cartesian product of the set of exposed points of the unit ball of $H^{1}$ with the set of inner functions.
\end{abstract}

\section{INTRODUCTION}

Let $S$ denote the unilateral shift on the Hardy space $H^{2}$ of the unit disk $D$. A subspace $M$ of $H^{2}$ is said to be nearly invariant under $S^{*}$ if $S^{*} f$ belongs to $M$ whenever $f$ belongs to $M$ and vanishes at the origin. Throughout this article, only closed subspaces will be considered. For an example, let $T_{\phi}$ denote Toeplitz operator on $H^{2}$ with symbol $\phi$ in $L^{\infty}$. Then $\operatorname{Ker} T_{\phi}$ is nearly invariant since $T_{\phi} S^{*}=S^{*} T_{\phi} S S^{*}$. The general notion of nearly invariant subspaces of $S^{*}$ was studied by D. Hitt [7] in connection with invariant subspaces of $H^{2}$ of an annulus. He provided the following description:

Theorem 1. Let $M$ be a proper nontrivial subspace of $H^{2}$ which is nearly invariant under $S^{*}$, and let $g$ be the function of unit norm in $M$ which is orthogonal to $M \cap H_{0}^{2}$ and is positive at the origin. Then $M=T_{g}\left(H^{2} \ominus u H^{2}\right)$ where $u$ is some inner function which vanishes at the origin. Furthermore, $T_{g}$ acts isometrically on the left invariant subspace $H^{2} \ominus u H^{2}$.

Using different methods, the author had independently obtained the above result in the special case that $M=\operatorname{Ker} T_{\phi}$ for any $\phi$ in $L^{\infty}$, in which case it was additionally shown that $g^{2}$ is an exposed point of the unit ball of the Hardy space $H^{1}$. Henceforth, such functions will be called exposed points of

Received by the editors July 31, 1989.

1980 Mathematics Subject Classification (1985 Revision). Primary 30D55, 43B35. 
$H^{1}$. It should be mentioned that if $M$ is nearly invariant and $v$ is any inner function that does not vanish at the origin, then $T_{v} M$ is also nearly invariant. So, in general, the function $g$ is outer only to the extent that is possible within $M$ : its inner part is the greatest common inner divisor of $M$.

Two natural questions that now come to mind are:

(1) Which pairs of functions $g$ and $u$ arise in connection with nearly invariant subspaces?

(2) Which pairs $g$ and $u$ arise in connection with the kernels of Toeplitz operators?

Sarason answered the first question in [6]. It is tempting to conjecture that the second question has the same answer as the first with the additional restriction that $g$ be an outer function whose square is an exposed point in $H^{1}$. After all, if $g$ is such a function and $T_{g}$ acts isometrically on $H^{2} \ominus u H^{2}$, then $T_{g}\left(H^{2} \ominus u H^{2}\right)$ is contained in $\operatorname{Ker} T_{\overline{u g} / g}$ and equality can be shown to hold when the latter space is of finite dimension. However, an example will be furnished in $\S 3$ to show that the above conjecture is not generally valid. $\S 2$ gives a summary of Sarason's insightful answer to question (1). This provides the main tools used throughout the rest of the paper. The answer to question (2) is given by Theorem 4 of $\S 4$, while Theorem 5 provides a parametrization of the kernels of Toeplitz operators. The main technical result used in establishing these theorems is given by the lemma of $\S 4$. It is proved by playing two extremal functions against each other.

\section{SARASON'S RESULTS}

Let $g$ be a function in $H^{2}$ having unit norm, and let $F$ denote the Herglotz integral of $|g|^{2}$, i.e., for $z$ in $D$,

$$
F(z)=\frac{1}{2 \pi} \int_{-\pi}^{\pi} \frac{e^{i \theta}+z}{e^{i \theta}-z}\left|g\left(e^{i \theta}\right)\right|^{2} d \theta .
$$

Then $F(0)=1$ and $F(z)$ has positive real part for $z$ in $D$. (The distinction between functions on the unit circle $\partial D$ and their Poisson extentions into $D$ will not be made explicit, since the appropriate interpretations are clear from context.) Thus, the function $b$ defined by $b=(F-1) /(F+1)$ lies in the unit ball of $H^{\infty}$ and vanishes at the origin. Now consider the Toeplitz operator $T_{g}$. If $g$ is not essentially bounded, $T_{g}$ is a densely defined unbounded operator. That $T_{g}$ can act isometrically as in Theorem 1 is somewhat suprising. This phenomenon was clarified by Sarason in the following theorem (see [6, 7]).

Theorem 2. Let $g$ and $b$ be as above and let $u$ denote an inner function with $u(0)=0$. Then $T_{g}$ acts isometrically on $H^{2} \ominus u H^{2}$ if and only if $u$ divides $b$ in $H^{\infty}$.

Note that any nontrivial nearly invariant subspace must contain a function which does not vanish at the origin. (Just apply suitably high powers of $S^{*}$.) 
So, in order to produce a candidate for a nearly invariant subspace, choose a function $g$ in $H^{2}$ which has unit norm but does not vanish at the origin. It does no harm to normalize $g$ to have a positive value at zero. Construct the function $b$ accordingly, and let $u$ be any inner function which divides $b$ and vanishes at zero. Then form $M=T_{g}\left(H^{2} \ominus u H^{2}\right)$. If $f$ is in $M$ and $f(0)=0$, we may write $f=g h$ where $h(0)=0$ and $h$ is in $H^{2} \ominus u H^{2}$. Thus $S^{*} f=g S^{*} h$ which is in $M$ as well. So $M$ is nearly invariant and, in view of the isometric property of $T_{g}$ guaranteed by Theorem 2, it is clear that $g$ and $u$ agree with their namesakes in Theorem 1 . Thus we have

Corollary. The proper nontrivial nearly invariant subspaces of $S^{*}$ are precisely those of the form $T_{g}\left(H^{2} \ominus u H^{2}\right)$, where $g$ belongs to $H^{2}$ having unit norm and positive value at the origin and where $u$ is an inner function which divides the inner part of the function $b$ related to $g$ as above with $u(0)=0$. Furthermore, $T_{g}$ acts isometrically on $H^{2} \ominus u H^{2}$.

\section{AN EXAMPLE}

Consider a Toeplitz operator $T_{\phi}$ with a nontrivial kernel. It was shown in $[3,4]$ that there exists a function $h$ in $H^{2}$ such that $\operatorname{Ker} T_{\phi}=\operatorname{Ker} T_{\overline{z h} / h}=$ $H^{2} \cap(h / \bar{h}) \bar{H}^{2}$. The following theorem was also proved:

Theorem 3. Let $h$ be a function in $H^{2}$ which is not identically zero, and let $M=\operatorname{Ker} T_{\overline{z h} / h}$. Let $g$ be the extremal function in the unit sphere of $M$ for the functional $f \rightarrow \operatorname{Re} f(0)$. Then there is an inner function $u$ which vanishes at the origin such that $z h / \bar{h}=u g / \bar{g}$ and $M=T_{g}\left(H^{2} \ominus u H^{2}\right)$ with $T_{g}$ acting isometrically on $H^{2} \ominus u H^{2}$. Furthermore, $g^{2}$ is an exposed point in $H^{1}$.

It is clear that the functions $g$ and $u$ above agree with the ones in Theorem 1. So the candidates for the kernels of Toeplitz operators are those subspaces of the form $T_{g}\left(H^{2} \ominus u H^{2}\right)$, where $g$ and $u$ are as in Theorem 2 with $g^{2}$ an exposed point of $H^{1}$. Moreover, Theorem 3 and the remarks which precede it imply that if such a candidate is actually the kernel of a Toeplitz operator, it must be the kernel of $T_{\overline{u g} / g}$.

Now consider the following example. Let $\left(r_{n}\right)$ denote a Blaschke sequence in $D$ consisting of real numbers tending to -1 , and let $v$ denote the corresponding Blaschke product. Also, let $u=z v, a=(1+z) / 2$, and $b=(1-z) u / 2$. Then $|a|^{2}+|b|^{2}=1$ a.e. on $\partial D, a(0)>0, a$ is outer, $b(0)=0$, and both $a$ and $b$ are in the unit ball of $H^{\infty}$. Let $g=a /(1-b)$. Then $g$ is an outer function in $H^{2}$ and is related to $b$ as in $\S 2$ (see [7]). Sarason studied this example and showed that $g^{2}$ is an exposed point of $H^{1}$ but that $1 / g$ is not in $H^{1}$. Note that $u$ divides $b$, so if $M=T_{g}\left(H^{2} \ominus u H^{2}\right)$, it is eligible to be the kernel of a Toeplitz operator. However,

$$
\overline{u g} / g=[2 \overline{u z}+\bar{z}-1] /[2+\bar{u}(1-\bar{z})],
$$


which is the complex conjugate of an inner function. Thus, if $M$ is to be the kernel of a Toeplitz operator, $M$ must be invariant under $S^{*}$, hence $S^{*} g$ is in $M$. Since multiplication by $1 / g$ is an isometry on $M$, this implies that $1 / g$ belongs to $H^{2}$, contradicting Sarason's result. Thus $M$ is not the kernel of any Toeplitz operator. As we shall see in the next section, this example points to the function theoretic properties which are special to the kernels of Toeplitz operators within the class of nearly invariant subspaces.

\section{Main Results}

Let $f$ be any outer function in the unit sphere of $H^{2}$ having a positive value at the origin, and let $F$ denote the Herglotz integral of $|f|^{2}$. Define $b=(F-1) /(F+1)$, and let $a$ denote the outer function with positive value at the origin such that $|a|^{2}+|b|^{2}=1$ a.e. on $D$. Then $a=2 f /(F+1)$ and $f=a /(1-b)$ (see [7]). If $v$ is any inner function, let $f_{v}$ denote the function $a /(1-v b)$. Then $f_{v}$ is related to $a$ and $v b$ in the same way $f$ is related to $a$ and $b$. Moreover, Sarason showed that $f_{v}^{2}$ is an exposed point of $H^{1}$ whenever $f^{2}$ is. In the example of the last section, $g=f_{v}$ where $f=a /(1-b)$, and $a, b$, and $v$ are as defined there. However, as was shown in [7], $f^{2}$ is not exposed. This suggests the following theorem whose proof is given at the end of this section.

Theorem 4. Let $M=T_{g}\left(H^{2} \ominus u H^{2}\right)$ as in Theorem 1. Then $M$ is the kernel of a Toeplitz operator if and only if $g$ is an outer function and

$$
\left(\frac{a}{(1-z \bar{u} b)}\right)^{2}
$$

is an exposed point in $H^{1}$, where $b$ is related to $g$ as in $\S 2$, and where $a$ is related to $b$ as above.

An immediate consequence of this is the following theorem which establishes a one to one correspondence between the kernels of Toeplitz operators and the Cartesian product of the set of exposed points in the unit ball of $H^{1}$ which are positive at 0 and the set of inner functions.

Theorem 5. The nontrivial kernels of Toeplitz operators are precisely those subspaces of $\mathrm{H}^{2}$ having the form

$$
M=T_{f_{v}}\left(H^{2} \ominus z v H^{2}\right),
$$

where $v$ is an inner function and $f$ is an outer function in $H^{2}$ which is positive at the origin and such $f^{2}$ is an exposed point of the unit ball of $H^{1}$. Moreover the functions $f$ and $v$ are uniquely determined by $M$.

To establish Theorem 4, it is necessary to consider the solutions of two extremal problems. The first involves the function $g$ in Theorem 1 . It is the unique function in $M$ which maximizes the functional $f \rightarrow \operatorname{Re} f(0)$ over the 
unit sphere of $M$. The second extremal function arises in connection with the Nehari interpolation problem described briefly below.

Let $w$ denote a function in $L^{\infty}$ of $\partial D$, and let $K(w)$ denote the set of elements in the coset $w+H^{\infty}$ which have norm at most one in $L^{\infty}$. The problem considered by Nehari was to find all functions in $K(w)$. He showed that $K(w)$ is nonempty if and only if the Hankel operator associated with $w$ is a contraction. In the indeterminate case that $K(w)$ has more than one element, Adamjan, Arov, and Krein [1] gave a parametrization of $K(w)$ as all functions of the form

$$
\frac{a}{\bar{a}} \frac{u-\bar{b}}{1-b u}
$$

where $u$ ranges over the unit ball of $H^{\infty}$ and where $a$ and $b$ are functions in the unit ball of $H^{\infty}$ satisfying the following properties: (i) $a$ is an outer function with $a(0)>0$, (ii) $b(0)=0$, and (iii) $|a|^{2}+|b|^{2}=1$ a.e. on $\partial D$. The pair $(a, b)$ is uniquely determined by $K(w)$ and will be called a Nehari pair after Sarason (see the discussion in [7]) and will also be denoted by $N(w)$. One may now form the function $f=a /(1-b)$. The second extremal function to be considered is $f / \bar{f}$ : it uniquely maximizes the functional $k \rightarrow \operatorname{Re} \int_{-\pi}^{\pi} k d \theta / 2 \pi$ over $K(w)$. This is most easily seen by noting that if $u$ is in the unit ball of $H^{\infty}$, then

$$
\frac{f}{\bar{f}}-\frac{a}{\bar{a}} \frac{u-\bar{b}}{1-b u}=\frac{a^{2}}{1-b} \frac{1-u}{1-u b}
$$

is in $H^{\infty}$ (as it should be) and its value at 0 has a nonnegative real part which vanishes if and only if $u$ is the constant 1 . This last extremal problem was considered by Garnett (see [2, p.160]) in an alternative derivation of the above parametrization.

Another fact that is needed is that if $(a, b)$ is any pair of functions in the unit ball of $H^{\infty}$ which satisfies (i)-(iii), then it is a Nehari pair for an indeterminate Nehari problem if and only if $[a /(1-b)]^{2}$ is an exposed point in $H^{1}$ (see [7]). Suppose now that $\phi$ is in $L^{\infty}$ such that $T_{\phi}$ has a nontrivial kernel. Write $\operatorname{Ker} T_{\phi}=\operatorname{Ker} T_{\overline{z h} / h}=T_{g_{0}}\left(H^{2} \ominus z u_{0} H^{2}\right)$ and $h / \bar{h}=u_{0} g_{0} / \bar{g}_{0}$ as in Theorem 3. Let $b_{0}$ and $g_{0}$ be related as in $\S 2$ and let $a_{0}$ and $b_{0}$ satisfy (i) and (iii) above. Then $g_{0}=a_{0} /\left(1-b_{0}\right)$ and Theorem 2 implies that $z u_{0}$ divides the inner part of $b_{0}$. Let $b_{1}=\bar{u}_{0} b_{0}$. Then $b_{1}$ is in the unit ball of $H^{\infty}$ with $\left|a_{0}\right|^{2}+\left|b_{1}\right|^{2}=1$ a.e. on $\partial D$. Also, $b_{1}(0)=0$ and

$$
\frac{h}{\bar{h}}=\frac{u_{0} g_{0}}{\bar{g}_{0}}=\frac{u_{0} a_{0}}{\bar{a}_{0}} \frac{1-\bar{b}_{0}}{1-b_{0}}=\frac{a_{0}}{\bar{a}_{0}} \frac{u_{0}-\bar{b}_{1}}{1-u_{0} b_{1}} .
$$

So if $\psi=h / \bar{h}-\left(a_{0} / \bar{a}_{0}\right) \cdot\left[\left(1-\bar{b}_{1}\right) /\left(1-b_{1}\right)\right]$, a simple computation shows that

$$
\psi=\frac{a_{0}^{2}\left(u_{0}-1\right)}{\left(1-b_{1}\right)\left(1-u_{0} b_{1}\right)},
$$


which is in $H^{\infty}$. Thus, in the case that $u_{0}$ is not identically $1, K(h / \bar{h})$ contains at least two functions. In the case that $u_{0}=1, h / \bar{h}=g_{0} / \bar{g}_{0}$. Since $g_{0}^{2}$ is an exposed point, $\left(a_{0}, b_{0}\right)$ is a Nehari pair. So, in either case, $N(h / \bar{h})$ may be defined.

Lemma. Let $h$ be a function in $H^{2}$ with

$$
\operatorname{Ker} T_{\overline{z h} / h}=T_{g_{0}}\left(H^{2} \ominus z u_{0} H^{2}\right)
$$

as above. Let $(a, b)=N(h / \bar{h})$, and let $u$ be defined by

$$
\frac{h}{\bar{h}}=\frac{a}{\bar{a}} \frac{u-\bar{b}}{1-b u}
$$

as in (3). Then $u$ is an inner function and the following hold:

(i) $g_{0}=f_{u}$ where $f=a /(1-b)$

(ii) $(a, b)=\left(a_{0}, \bar{u}_{0} b_{0}\right)$, where $a_{0}$ and $b_{0}$ are as above.

Proof. Let $h, g_{0}, u_{0}, a_{0}$, and $b_{0}$ be defined as in the paragraph preceding the lemma, and let $(a, b)=N(h / \bar{h})$. Then $h / \bar{h}=(a / \bar{a})[(u-\bar{b}) /(1-u b)]$ for some $u$ as in (3), and since $h / \bar{h}$ has unit modulus a.e. on $\partial D$, it follows that $u$ is an inner function. Factoring $u$ out of the right-hand expression yields $h / \bar{h}=u f_{u} / \bar{f}_{u}$ where $f=a /(1-b)$. It is easily checked that $f_{u}$ is in $H^{2} \cap(h / \bar{h}) \bar{H}^{2}=\operatorname{Ker} T_{\overline{z h} / h}$. Sarason's analysis shows that $f_{u}$ has unit norm in $H^{2}$ so it follows from the extremal property of $g_{0}$ that

$$
a(0)=f_{u}(0) \leq g_{0}(0)=a_{0}(0) .
$$

By the way $\psi$ is defined, it follows from (4) that $\left(a_{0} / \bar{a}_{0}\right)\left[\left(1-\bar{b}_{1}\right) /\left(1-b_{1}\right)\right]$ is in $K(h / \bar{h})$ and that

$$
\begin{aligned}
\int_{-\pi}^{\pi} \frac{a_{0}}{\bar{a}_{0}} \frac{1-\bar{b}_{1}}{1-b_{1}} d \theta / 2 \pi & =\int_{-\pi}^{\pi}\left(\frac{h}{\bar{h}}-\psi\right) d \theta / 2 \pi \\
& =\lambda_{0}+a_{0}(0)^{2}\left[1-u_{0}(0)\right],
\end{aligned}
$$

where

$$
\lambda_{0}=\int_{-\pi}^{\pi}(h / \bar{h}) d \theta / 2 \pi
$$

Since $f / \bar{f}=h / \bar{h}+(1-u) a^{2} /[(1-b)(1-u b)]$, we have

$$
\int_{-\pi}^{\pi}(f / \bar{f}) d \theta / 2 \pi=\lambda_{0}+a(0)^{2}[1-u(0)] .
$$

Equations (6) and (7) together with the extremal property of $f / \bar{f}$ imply that

$$
\operatorname{Re}\left[a_{0}(0)^{2}\left(1-u_{0}(0)\right)\right] \leq \operatorname{Re}\left[a(0)^{2}(1-u(0))\right] .
$$

In view of $(5)$ and the reality of $a_{0}(0)$ and $a(0)$, we then have

$$
a_{0}(0)^{2} \operatorname{Re}\left(1-u_{0}(0)\right) \leq a(0)^{2} \operatorname{Re}(1-u(0)) \leq a_{0}(0)^{2} \operatorname{Re}(1-u(0)) .
$$


For any fixed angle $\theta$, a simple algebraic manipulation shows that

$$
N\left(e^{i \theta} h / \bar{h}\right)=\left(a, e^{-i \theta} b\right) .
$$

Furthermore,

$$
e^{i \theta} h / \bar{h}=\frac{a}{\bar{a}} \frac{e^{i \theta} u-\bar{b}_{\theta}}{1-e^{i \theta} u b_{\theta}}
$$

where $b_{\theta}=e^{-i \theta} b$. Using the argument leading to (8), we have, for all $\theta$,

$$
a_{0}(0)^{2} \operatorname{Re}\left(1-e^{i \theta} u_{0}(0)\right) \leq a(0)^{2} \operatorname{Re}\left(1-e^{i \theta} u(0)\right) \leq a_{0}(0)^{2} \operatorname{Re}\left(1-e^{i \theta} u(0)\right) \text {. }
$$

Thus, $\operatorname{Re}\left(e^{i \theta}\left(u_{0}(0)-u(0)\right) \geq 0\right.$ for all $\theta$, so $u_{0}(0)=u(0)$. In the case that the common value is unity, both $u$ and $u_{0}$ must be constant, so $g_{0} / \bar{g}_{0}=$ $h / \bar{h}=f / \bar{f}$. Because $f^{2}$ is exposed, $g_{0}=f$ so (i) and (ii) follow. In the other case, it follows from (8) that $a_{0}(0)=a(0)$, so $g_{0}(0)=f_{u}(0)$. The extremal property of $g_{0}$ now implies that $f_{u}=g_{0}$, so $a=a_{0}$ and $u b=b_{0}$. Also, $u_{0} g_{0} / \bar{g}_{0}=h / \bar{h}=u f_{u} / \bar{f}_{u}$, so $u=u_{0}$. Thus, $b=\bar{u} b_{0}=\bar{u}_{0} b_{0}$. This in turn implies that

$$
N(h / \bar{h})=(a, b)=\left(a_{0}, \bar{u}_{0} b_{0}\right)
$$

and that $\left[a_{0} /\left(1-\bar{u}_{0} b_{0}\right)\right]^{2}$ is an exposed point of $H^{1}$. This concludes the proof of the lemma.

Proof of Theorem 4. Let $M=T_{g}\left(H^{2} \ominus u H^{2}\right)$ be as stated in the hypothesis of Theorem 4. If $M$ is the kernel of a Toeplitz operator, then, by Theorem 3, $M=$ $\operatorname{Ker} T_{\overline{z h} / h}$ with $z h / \bar{h}=u g / \bar{g}$. Making the identifications $u=z u_{0}$ and $g=g_{0}$, it follows from the lemma that $N(h / \bar{h})=(a, z \bar{u} b)$ so that $[a /(1-z \bar{u} b)]^{2}$ is an exposed point of $H^{1}$.

Conversely, suppose $M=T_{g}\left(H^{2} \ominus u H^{2}\right)$ as in Theorem 1 and that $g$ is an outer function with $[a /(1-z \bar{u} b)]^{2}$ exposed in $H^{1}$. Let $f=a /(1-z \bar{u} b)$ and $y=\overline{u g} / g$. Then

$$
\operatorname{Ker} T_{y} \supseteq T_{g}\left(H^{2} \ominus u H^{2}\right) .
$$

We may also write $u g / \bar{g}=z u_{0} g_{0} / \bar{g}_{0}$ as in the lemma. We need to show that $u=z u_{0}$ and $g=g_{0}$. Following the notation of the lemma, $\left(a_{0}, \bar{u}_{0} b_{0}\right)=$ $N\left(u_{0} g_{0} / \bar{g}_{0}\right)$. Because $f^{2}$ is exposed, $(a, z \bar{u} b)$ is a Nehari pair. Now note that

$$
\begin{aligned}
u_{0} g_{0} / \bar{g}_{0} & =\bar{z} u g / \bar{g} \\
& =\bar{z} u \frac{a}{\bar{a}} \frac{1-\bar{b}}{1-b} \\
& =\frac{a}{\bar{a}} \frac{\bar{z} u-\bar{z} u \bar{b}}{1-(\bar{z} u)(z \bar{u} b)} .
\end{aligned}
$$

This last function belongs to the coset of $H^{\infty}$ associated with the Nehari pair $(a, z \bar{u} b)$. Since distinct cosets are disjoint and Nehari pairs are unique, it 
follows that

$$
(a, z \bar{u} b)=N\left(u_{0} g_{0} / \bar{g}_{0}\right)=\left(a_{0}, \bar{u}_{0} b_{0}\right) .
$$

Additionally, (10) and the lemma imply that $g_{0}=f_{v}$ with $v=\bar{z} u$. But $f_{v}=g$, so $g_{0}=g$ and hence $b=b_{0}$. It now follows from (11) that $u=z u_{0}$, so equality must hold in (9). This concludes the proof of Theorem 4.

\section{REFERENCES}

1. V. M. Adamjan, D. Z. Arov and M. G. Krein, Infinite Hankel matrices and generalized problems of Carathéodory-Fejer and I. Schur, Funkcional. Anal. i Priloźen 2 (1968), 1-17.

. 2. J. B. Garnett, Bounded analytic functions, Academic Press, New York, 1981.

3. E. Hayashi, The solution sets of extremal problems in $H^{1}$, Proc. Amer. Math. Soc. 93 (1985), 690-696.

4. _ The kernel of a Toeplitz operator, Integral Equations Operator Theory 9 (1986), 588-591.

5. D. Hitt, Invariant subspaces of $H^{2}$ of an annulus, preprint.

6. D. Sarason, Nearly invariant subspaces of the backward shift, Oper. Theory: Adv. Appl. 35 (1988), 481-493.

7. __ Exposed points in $H^{1}$ I, Oper. Theory: Adv. Appl. (to appear).

Department of Mathematics, San Francisco State University, San Francisco, CaliFORNIA 94132 